\title{
On Positron Radiation Belt in the Earth's Magnetosphere
}

\author{
A.A. Gusev ${ }^{1}$, U.B. Jayanthi ${ }^{2}$, I.M. Martin ${ }^{1}$, G.I.Pugacheva ${ }^{1}$, and W.N. Spjeldik ${ }^{3}$ \\ ${ }^{1}$ University of Campinas, Instituto de Física/DRCC, 13083-970, Campinas, SP, Brazil \\ ${ }^{2}$ Instituto Nacional das Pesquisas Espaciais, INPE, São José dos Campos, SP, Brazil \\ ${ }^{3}$ Department of Physics, Weber State University, Ogden, Utah 84408-2508, USA
}

Received 28 February, 2000

\begin{abstract}
The ratio of positron/electron fluxes originated in nuclear spallation reactions in the Earth's magnetosphere is considered. It is supposed that positrons as well as electrons are produced in the decay of charged pions $\left(\pi^{ \pm} \rightarrow \mu^{ \pm} \rightarrow e^{ \pm}\right)$born in nuclear collisions of trapped relativistic inner zone protons with the residual atmosphere. These positrons and electrons are captured in the magnetosphere and create positron and electron radiation belts of nuclear origin. The positron/electron trapped magnetospheric fluxes formed with this mechanism are simulated and the resulting computed $e^{+} / e^{-}$ flux ratio $\approx 4$ appears in agreement with the recent observations. This ratio is significantly different from the ratio $\approx 1$ obtained from the primary cosmic ray source through the same mechanism.
\end{abstract}

\section{Introduction}

In our recent papers $[1,2]$ we showed that significant fluxes of trapped anti-particles must exist in the innermost magnetosphere of the Earth. However they are not of that primordial nature of origin, which attracts the general interest of astrophysics community, but are born in a more trivial process, as secondaries from nuclear spallation reactions of energetic trapped protons and primary cosmic rays (CR) with the residual terrestrial (or other planet's, for example, Jovian) atmosphere. These secondary particles being trapped by the Earth's magnetic field can be accumulated there and form more radiation belts, for example, anti-proton and positron radiation belts, in addition to already existing ones i.e. those of protons, electrons and anomalous CR nuclei. These trapped fluxes of anti-protons and positrons could be even greater than the secondary interstellar fluxes which are generated by CR on only 5 - 7 g.cm ${ }^{2}$ of interstellar matter. These fluxes are produced on the 2-3 radiation lengths of electrons in the air [3], that is $\approx 36 \times 3=108 \mathrm{~g} / \mathrm{cm}^{2}$. Thus, they could be about 15 -20 times greater than interstellar ones at certain energies. During geomagnetic unstable periods they could possibly precipitate into the atmosphere or could leave the magnetosphere and appear in the interplanetary medium, causing new puzzles.

In the same spallation reactions the trapped energetic protons also produce the isotopes of light elements, like D, T, ${ }^{3} \mathrm{He}[4,5]$. The trapped deuterium and ${ }^{3} \mathrm{He}$ isotopes were actually observed recently by SAMPEX and CRRES satellites [6,7]. This discovery supports the idea that significant fluxes of trapped secondary positrons could be produced by the trapped protons on the rarefied density of the residual atmosphere at the altitudes of $300-1000 \mathrm{~km}$.

Significant trapped energetic positron fluxes could be observed according to this idea in the innerest part of the geomagnetosphere where atmosphere is rare, but still exists and where a sufficient flux of energetic protons exists to sustain the positron population.

However, until last year there was no clear experimental confirmation of the existence of a positron radiation belt. In the previous experiments with magnetic spectrometer performed on board of the COSMOS-1669 and MIR station contradictory results were obtained. It seems that the new generation of experiments finally managed to prove the idea: In July's 1999 issue of CERN Courier it was communicated that Alpha Magnetic Spectrometer (AMS) [8] observed high energy (> $200 \mathrm{MeV}$ ) positron flux in the equatorial region at the altitude of $400 \mathrm{~km}$ with the intensity about 4 times higher than the electron flux of the same energv. The authors characterized the result as very surprising and puzzling. However, we think that this observation is a confirmation of the above formulated mechanism which has been developed since 1982 [3,9].

In this paper it will be demonstrated that the observed positron excess is a crucial argument in favor of our idea. 


\section{The source of positrons}

From a theoretical point of view, the positron radiation belt generation is a bit more complicated than the formation of the isotope radiation belts. The geomagnetically confined positrons do not appear as an immediate direct product of a nuclear reaction. Rather, the basic mechanism of positron and electron nuclear collision generation is the muon-positron decay of charged positive pions and kaons, resulting from the same nuclear reactions where deuterium, tritium and ${ }^{3} \mathrm{He}$ secondary nuclei are born. The other difference is that for pion production, there exists an important minimum energy threshold of $290 \mathrm{MeV}$ per incident proton on an exospheric neutral. This large incident proton energy is necessary for a proton to produce a pion in the reaction with the atmospheric target nucleus.

To be specific, in nuclear spallation reactions both negative and positive pions and kaons will be produced along with the secondary nuclei. Once these short-lived particles are born they decay into:

$$
\pi^{ \pm}, K^{ \pm} \Rightarrow \mu^{ \pm}+\nu \quad \mu^{ \pm} \Rightarrow e^{ \pm}+\tilde{\nu}+\nu
$$

In fact, they are so short-lived that they decay practically at the same place in the magnetosphere where they are born. Their short lifetime $\tau_{0}$ is of 26 nanoseconds for charged pions and $2.197 \mu$ s for muons. They could travel from the point of generation for only the distance of about: $\gamma \tau_{0} v$, here $v$-pion's, muon's velocity, $\gamma$ is a particle Lorentz factor. For example, for 1000 $\mathrm{MeV}$ pion this travel distance is: $1000 / 139.57^{*} 2.610^{-8}$ $\mathrm{s} * 310^{5} \mathrm{~km} / \mathrm{s}=56 \mathrm{~m}$. For a $1000 \mathrm{MeV}$ muon the travel distance is: $1000 / 105.66 * 2.19710^{-6} \mathrm{~s} * 3.10^{5} \mathrm{~km} / \mathrm{s}=$ $6.25 \mathrm{~km}$. Thus, from the point of view of magnetospheric particle capture, it means that the positrons are born and trapped in the same region of magnetosphere, where energetic trapped protons exist. The confinement area at $L<1.2$ is concentrated in a very restricted angle near the geomagnetic equatorial plane. Due to that only the positrons born with velocity direction within this angle (i.e. near the plane of the parent proton Larmour rotation) can be captured and will be taken into account in the following consideration.

In Fig.1 we depict the location of $>400 \mathrm{MeV}$ trapped proton flux at the altitude of $400 \mathrm{~km}$ over atmosphere based on the AP-8 magnetospheric proton model [10].

Recognizing, however, the strong dynamics of the electron radiation belt observed recently [11], the positron belt spatial location may sometimes be different from that of the parent protons and the positron fluxes could even disappear from the area and appear in the interplanetary medium as the result of high geomagnetic activity.

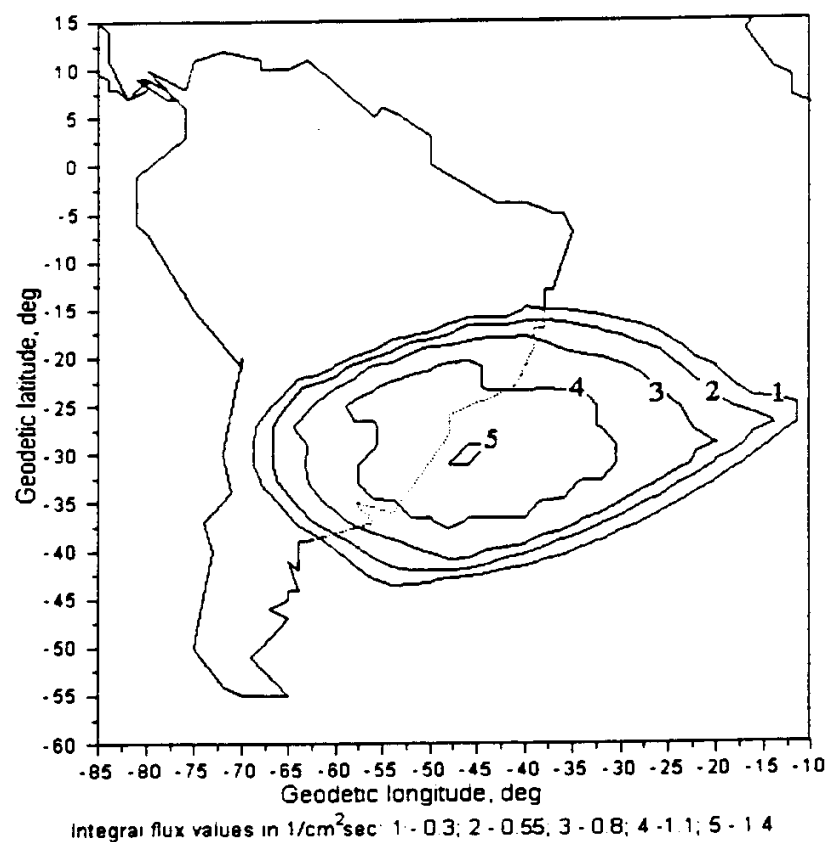

Figure 1. The space distribution of the $>400 \mathrm{MeV}$ trapped protons at the altitudes of $400 \mathrm{~km}$ according to AP-8 model (Vette, 1991).

\section{The positron belt formation}

To calculate the positron flux $F_{e^{+}}(E)$ we must integrate the positron differential production spectrum $P_{e^{ \pm}}\left(E^{\prime}\right)$ (i.e. fluxes generated in $1 \mathrm{~g} / \mathrm{cm}^{2}$ of the air) along the positron trajectory taking into account that a positron of energy $E$ was born with energy $E^{\prime}$ at the distance of $X^{\prime} \mathrm{g} / \mathrm{cm}^{2}$ from the point of observation. If the positron energy losses are $(d E / d X)$, it results in the relationship: $F_{e^{ \pm}}(E)=\int_{0}^{\infty} P_{e^{ \pm}}\left(E^{\prime}\right)\left(d E^{\prime} / d E\right) d X^{\prime}=P_{e^{ \pm}}(>$ $E) /(d E / d X)[2]$, i.e. the $e^{+} / e^{-}$flux ratio is proportional to the ratio of their integral production spectra.

The mean fraction of energy carried away by a muon in a pion's decay and by an electron in a muon decay is typically equal to $q_{1}=0.80$ and $q_{2}=0.33$ respectively. With this simplification, we can relate the positron (electron) $P_{e^{ \pm}}$and pion production spectra $Q_{\pi^{ \pm}}$with the following rather simple expression:

$$
P_{e \pm}(E)=\frac{1}{q_{1} q_{2}} Q_{\pi^{ \pm}}\left(E / q_{1} q_{2}\right) .
$$

From this set of physical ideas and a modest mathematical construction one can rather transparently see that positron/electron flux ratio at equal energies per particle is proportional to the ratio of positive and negative integral pion production spectra $Q_{\pi^{ \pm}}(>E)$ at the $l / q_{l} q_{2}$ greater energies than electron ones:

$$
F_{e^{+}}(E) / F_{e^{-}}(E)=\frac{Q_{\pi^{+}}\left(>E / q_{1} q_{2}\right)}{Q_{\pi^{-}}\left(>E / q_{1} q_{2}\right)} .
$$


The pion production spectra for $\pi^{ \pm}$pions were earlier computed [1] for trapped proton fluxes at $L=1.2$. These are presented in Table 1. From this one can see that this source mechanism provides four times greater positive pion fluxes than negative pion fluxes at pion energies of more than $600-800 \mathrm{MeV}$. Consequently, these production spectra of secondary particle fluxes can supply four times greater positron fluxes than electron fluxes at energies more than $150-200 \mathrm{MeV}$ on these geomagnetic $L$-shells. Indeed, this is precisely the same positron-to-electron flux ratio that was observed in the AMS experiment noted above for positron/electron flux ratio at energies of more than $200 \mathrm{MeV}$ per particle. We thus conclude that the puzzle appears resolved, and that there exists a simple physical explanation based on the knowledge of the nuclear collision reactions from which most positive pions are born.

Table 1. The pion production spectra.

\begin{tabular}{|c|c|c|c|}
\hline$e^{+}, e^{-}$energy & Pion energy & $Q_{\pi^{-}}$ & $Q_{\pi^{+}}$ \\
\hline $\mathrm{MeV}$ & $\mathrm{MeV}$ & $\mathrm{m}^{-2} \mathrm{~s}^{-1} \mathrm{sr}^{-1} \mathrm{GeV}^{-1} \mathrm{~g}^{-1} \mathrm{~cm}^{2}$ \\
\hline $0-5$ & $0-20$ & 556.4 & 760.5 \\
$5-10$ & $20-40$ & 750.6 & 1177.3 \\
$10-15$ & $40-60$ & 846.9 & 1359.3 \\
$15-20$ & $60-80$ & 763.7 & 1408.1 \\
$20-25$ & $80-100$ & 635.2 & 1072.7 \\
$25-50$ & $100-200$ & 411.4 & 1103.8 \\
$50-100$ & $200-400$ & 522.1 & 1596.5 \\
$100-150$ & $400-600$ & 172.6 & 540.1 \\
$150-200$ & $600-800$ & 70.0 & 301.2 \\
$200-250$ & $800-1000$ & 20.0 & 79.0 \\
\hline
\end{tabular}

The geomagnetically trapped protons have relatively low energies (from a nuclear interaction point of view) and less than 1 secondary pion per reaction is generated. Certainly they have positive (!) charge due to the charge conservation law. In comparison, in the case of positrons born by primary $\mathrm{CR}$, having tens $\mathrm{GeV}$ energies, a greater multiplicity of pions generated, and the positron flux excess is negligible.

To theoretically search a positron flux from the CR source, we used a version of a nuclear reaction computer code SHIELD for Monte Carlo simulation of hadron cascades in matter [12]. Using this code we computed positive and negative pion production spectra from protons with various energies corresponding to the uppermost range of trapped proton energies (500 MeV - 2 $\mathrm{GeV}$ ) in the innermost parts of the magnetosphere and to $\mathrm{CR}$ proton component (more than $8-10 \mathrm{GeV}$ for the magnetospheric equatorial region). The ratios of the integral production spectra of positive and negative pions for various parent proton energies are plotted in Fig. 2. As we mentioned above this ratio is the same as $e^{+} / e^{-}$flux ratio. From this figure one can immediately ascertain that the ratio of pion spectra from $\mathrm{CR}$ source is less than from the trapped proton source and equal $\sim 1$ for more than $100 \mathrm{MeV}$ positron energies.

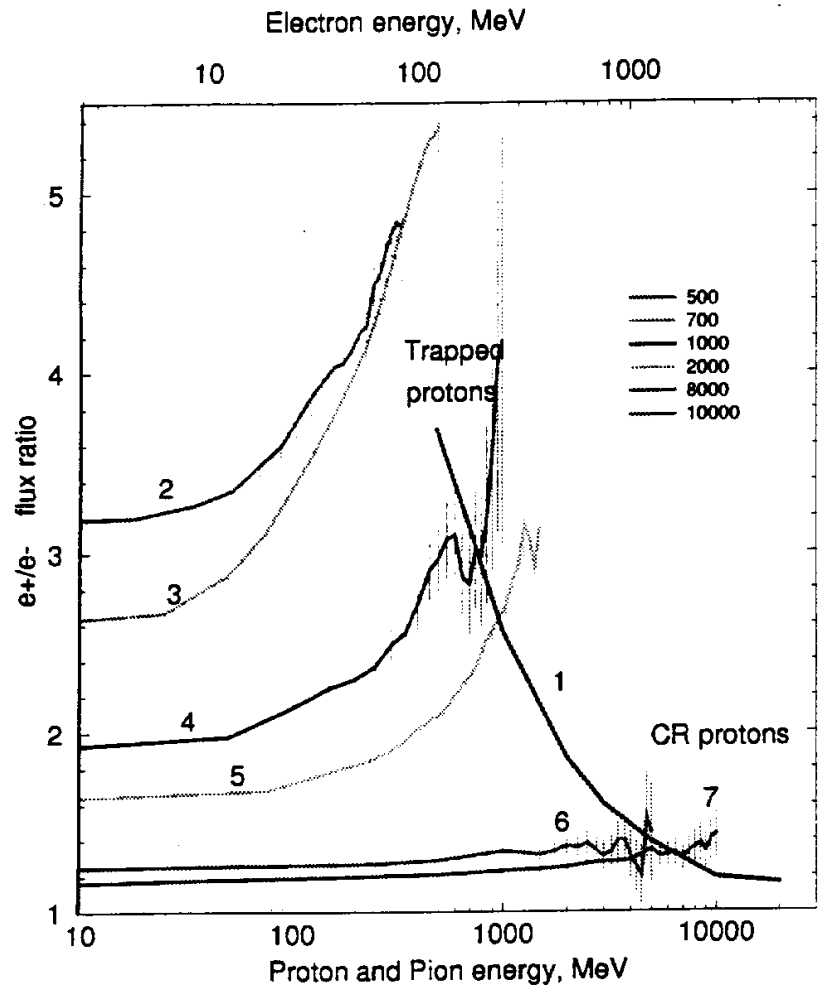

Figure 2. Curve 1: The dependence of the $e^{+} / e^{-}$-flux ratio on the parent proton energy; curves $2-7$ : The dependence of the $\pi^{+} / \pi^{-}$integral production flux ratio and $e^{+} / e^{-}$flux ratio on the $\pi$ and $e^{ \pm}$energies, correspondingly.

In the innermost part of magnetosphere, at low $L$ shells of $L \leq 1.2$ it is difficult to expect an existence of hundreds $\mathrm{MeV}$ trapped electrons of diffusive origin. As it was shown in $[13,14]$ the electrons can not diffuse there from the boundary of magnetosphere because they loss its energy due to synchrotron radiation and undergo strong pitch-angle scattering with plasma waves at the region of $L \approx 3$ on the way into inner magnetosphere. Thus, a diffusive electron component at those energies does not decrease computed $e^{+} / e^{-}$flux ratio obtained from nuclear source and in observations in the innermost part of magnetosphere exactly $e^{+} / e^{-}$ flux ratio equal to $\approx 4$ is expected to be registered in the case of nuclear reaction source of magnetospheric positrons.

\section{Conclusion}

A mechanism for generation of the terrestrial positron radiation belt due to nuclear reactions of trapped radiation belt protons with the nuclei of the neutral atoms in the upper atmosphere/exosphere region is considered. The ratio of positron/electron fluxes computed based on the in-situ collisional mechanism shows that the positron flux is expected to exceed almost exactly 
four times the ambient electron flux in the equatorial region for particles of more than $200 \mathrm{MeV}$. That is in excellent agreement with the AMS-instrument observations reported in the literature. In contrast, the CR source in this region provides a positron/electron flux ratio about equal 1 . The $\mathrm{CR}$ fluxes are thus not responsible for the observed innermost radiation belt positrons and electrons. We believe that this physical consideration will help to solve the puzzle of the unique AMS positron belt observation.

\section{Acknowledgements}

The work was supported by FAPESP-SP (93/49780) and $\mathrm{CNPq}_{(300015 / 99-2) \text {. }}$

\section{References}

[1] A.A. Gusev, I.M. Martin, G.I. Pugacheva, A. Turtelli Jr., W.N. Spjeldvik, Il Nuovo Cimento, Sect. C, 19, 461 (1996).

[2] G.I. Pugacheva, W.N. Spjeldvik, A.A. Gusev, I.M. Martin, J. Atm. Sol.-Terr. Phys. 59, 363 (1997).

[3] R.N. Basilova, A.A. Gusev, G.I. Pugacheva, A.F. Titenkov, Geomagnetism and Aeronomia, 22, 671 (1982).

[4] W.N. Spjeldvik, G.I. Pugacheva, I.M. Martin, A.A. Gusev, N.M. Sobolevsky, Advances in Space Research, 21, 1675 (1998)a.
[5] W.N. Spjeldvik, G.I. Pugacheva, A.A. Gusev, I.M. Martin, and N.M. Sobolevsky, Annales Geophysicae, 16, 931 (1998)b.

[6] J. Chen, T.G. Guzik, J.P. Wefel, K.R. Pyle, and J.F. Cooper, J. Geophys. Res. 101, 24 (1996).

[7] R.S. Selesnick, and R.A. Mewaldt, J. Geophys. Res. 101, 19745 (1996).

[8] Cern Courier, Magnetic detector sees cosmic-ray anomalies, 39, 6 (1999).

[9] I.M. Martin, A.A. Gusev, G.I. Pugacheva, A. Turtelli Jr., Yu.V. Mineev, J.Atmosph. and Terr. Phys. 57.2, 201 (1995).

[10] J.I. Vette, Rep. NSSDC 91-24, NASA Goddard Space Flight Center, Greenbelt, Md. November, (1991).

[11] A.A. Gusev, T. Kohno, I.M. Martin, G.I. Pugacheva, A. Turtelli Jr., A. Tylka, K. Kudela. Planetary and Space Science, 43, 1131 (1995).

[12] N.M. Sobolevsky, G.I. Pugacheva, A.A. Gusev, and I.M. Martin, The-Proceedings of the 8th International Conference on Radiation Shielding, April 24-28, 1994: Arlington, TX, published by the American Nuclear Society, 619 (1994).

[13] G.I. Pugacheva, D.M. Boscher, A.A. Gusev, I.M. Martin, W.N. Spjeldvik, Geoph. Res. Let. 25, 1519 (1998)a.

[14] G.I. Pugacheva, D.M. Boscher, A.A. Gusev, I.M. Martin, W N. Spjeldvik, J. Atm. Sol.-Terr. Phys. 60, 1159 (1998)b. 